\title{
DOS PARÁSITOS BRANQUIALES DE LA CACHEMA Cynoscion analis JENYNS 1842 (OSTEICHTHYES: SCIAENIDAE) DE PERÚ
}

\author{
José Iannacone ${ }^{1}$
}

\begin{abstract}
RESUMEN
Ciento veinte especímenes de Cachema Cynoscion analis Jenyns (Pisces: Sciaenidae) fueron colectados del Terminal Pesquero de Chorrillos, Lima, Perú, entre Enero a Febrero del 2000 y necropsiados para el estudio del monogeneo ectoparásito Cynoscionicola cynoscioni Tantalean, Martinez \& Escalante, 1987 y del copépodo Lernanthropus paralonchuri Luque, Bruno \& Covarrubias, 1989 de los filamentos branquiales. La prevalencia de infección de C. cynoscioni fue $21,70 \%$, la intensidad y la abundancia media fueron 1,84 $\pm 2,38$ y $0,39 \pm 1,32$, respectivamente. Este parasito tuvo una distribución poblacional espacial sobredispersa $(3,13)$, entre los hospederos evaluados. L. paralonchuri tuvo $16,70 \%$ de prevalencia, $1,26 \pm 0,73$ de intensidad y $0,20 \pm 0,54$ de abundancia media de infestación. Este parasito no tuvo una distribución poblacional espacial sobredispersa $(0,42)$ entre los hospederos evaluados. En general, se observó una carencia de relación entre la prevalencia, intensidad y abundancia media de ambos ectoparasitos con la longitud y el sexo de $C$. analis. Solo $C$. cynoscioni mostró una relación positiva entre la abundancia media y el tamaño corporal del pez y además $L$. paralonchuri tuvo la misma relación entre la prevalencia y la intensidad media con la longitud. El tamaño corporal de los peces parasitados y no parasitados con $C$. cynoscioni mostró diferencias significativas. La mayor prevalencia y el promedio de abundancia de $C$. cynoscioni y $L$. paralonchuri se encontró en el I y IV par branquial, respectivamente. Ambos parasitos no mostraron preferencias a los 24 sectores branquiales (anterior, medio y posterior).
\end{abstract}

Palabras claves: Cynoscion, copepodo, Cynoscionicola, Lernanthropus, monogenea.

\section{SUMMARY}

One hundred and twenty specimens of Common Peruvian weakfish Cynoscion analis Jenyns 1842 (Pisces: Sciaenidae) were collected from Chorrillos fishmarket, Lima, Perú, between January and February 2000 and necropsied to study ectoparasite monogenean Cynoscionicola cynoscioni Tantalean, Martinez \& Escalante, 1987 and the copepod Lernanthropus paralonchuri Luque, Bruno \& Covarrubias, 1989 from gill filaments. The prevalence of infection of C. cynoscioni was $21.70 \%$, mean intensity and abundance were $1.84 \pm 2.38$ and $0.39 \pm 1.32$, respectively. This parasite had an overdispersal spatial population (3.13) between hosts evaluated. Lernanthropus paralonchuri had a prevalence, mean intensity and abundance of infection $16.70 \%, 1.26 \pm 0.73$ and $0.20 \pm 0.54$. This parasite did not have an overdispersal spatial population (0.42) between hosts evaluated. In general, a

\footnotetext{
Laboratorio de Invertebrados. Facultad de Ciencias Biológicas. Universidad Ricardo Palma, mail:jiannacone@mail.urp.edu.pe
} 
lack of relation among prevalence, mean intensity and abundance of both parasites with length and sex of $C$. analis was observed. Only $C$. cynoscioni showed a positive relationship between mean abundance and fish body size and therefore L. paralonchuri had the same relationship between prevalence and mean intensity with length. Fish body size among parasited and not parasited with $C$. cynoscioni found differences significantly. The highest prevalence and mean abundance of $C$. cynoscioni and $L$. paralonchuri were on I and IV pairs of gills, respectively. Both parasites not showed preferences to 24 gill sectors (fore, middle and hind).

Key words: Cynoscion, copepod, cynoscionicola, lernanthropus, monogenea.

\section{INTRODUCCIÓN}

Cynoscion analis Jenyns 1842, «cachema»o «Ayanque» es un esciánido de importancia en las cadenas tróficas marinas en la costa central del Perú (Albitres, 1965). Su desembarque nacional se encuentra entre las diez especies de mayor captura total (Estrella et al., 1997). Su distribución geográfica conocida, va desde Perú a Chile (Mendo et al., 1988; Chirichigno \& Velez, 1998).

Se han publicado trabajos sobre la fauna parasitaria en peces Sciaenidae (Osteichthyes) de la Costa Peruana como C. analis, Menticirrhus ophicephalus (Jenyns 1842), Paralonchurus peruanus (Steindachner 1875), Sciaena deliciosa (Tschudi 1844), Sciaena fasciata (Tschudi 1845) y Stellifer minor (Tschudi 1844), estableciendo patrones básicos de las comunidades parasitarias con relación al tamaño de los hospedadores, sexo, variación estacional y estructura de las comunidades parasitarias (Saldarriaga, 1977; Oliva et al., 1989, 1990; Iannacone, 1991; Luque et al., 1991; Luque \& Oliva, 1993; Luque, 1994, 1996; Iannacone et al., 2000b c; Iannacone, 2004).

Cynoscionicola y Lernanthropus han sido registrados para el Perú parasitando a Sciaenidae y Carangidae. Algunas relaciones ecológicas han sido realizadas con sus peces hospederos (Iannacone, 1991; Luque, 1994; Oliva \& Luque, 1998). El monogeneo Cynoscionicola cynoscioni Tantalean, Martinez \& Escalante, 1987 ha sido registrado en $C$. analis por Iannacone et al. (2000c) y Lernanthropus paralonchuri Luque, Bruno \& Covarrubias, 1989 ha sido registrado en $P$. peruanus (Oliva \& Luque, 1998), y recientemente por Iannacone et al. (2000c) en C. analis.
La longitud estándar ha sido considerada importante por numerosos autores en estudios de ecología parasitaria (George-Nascimento \& Iriarte, 1989; Hayward et al., 1998; Iannacone et al., 2000a).

Este trabajo representa un primer análisis cuantitativo de las poblaciones de $C$. cynoscioni y de $L$. paralonchuri en las branquias de $C$. analis, con el objetivo de evaluar (1) la influencia del tamaño y sexo del hospedero sobre estos dos parásitos y (2) su posible distribución espacial de ambos parásitos al nivel de los arcos branquiales.

\section{MATERIAL Y MÉTODOS}

\section{Colección del Material Biológico}

Se adquirieron 120 especimenes de «Cachema», $C$. analis entre Enero y Febrero del 2000 en el Terminal Pesquero de Chorrillos, Perú $\left(12^{0} 30 \mathrm{~S}, 76^{0}\right.$ $50 \mathrm{E}$ ). Los peces se llevaron al laboratorio tratando que estos se mantuvieran en óptimo estado de conservación. Cynoscionicola cynoscioni y $L$. paralonchuri fueron colectados de los arcos branquiales. Lernanthropus paralonchuri fue sexado y separado en machos y hembras. En el laboratorio, se procedió a la extracción de los opérculos, los arcos branquiales, examinándolos en orden, los que fueron divididos en tres secciones $\mathrm{a}, \mathrm{b}, \mathrm{c}$ (anterior, medio $\mathrm{y}$ posterior respectivamente) siendo colocados en placas Petri con solución salina al $0,85 \%$ para el desprendimiento de la mucosidad y de los ectoparásitos de C. cynoscioni y L. paralonchuri. Esto se llevó a cabo siguiendo el protocolo de Kritsky et al. (1986) y de Williams (1989). 
Se determinó en los hospederos el sexo y la longitud estándar (cm). Además se registro el número y lugar de infestación de ambos ectoparásitos por pez hospedero (arcos branquiales $\mathrm{y}$ su respectiva sección).

Los monogeneos fueron limpiado, coloreados con carmín acético Semichon's diluido con alcohol al $70 \%$ (6:1) por 5'. Luego fue sometido a una batería de alcoholes $(70,85,95$ y $99 \%)$, finalmente fue montado en medio de Bálsamo de Canadá. El copépodo siguió el procedimiento descrito por Luque \& Farfán (1991).

Especimenes representativos fueron depositados en la Colección Helmintológica y de Invertebrados menores del Museo de Historia Natural de la Universidad Nacional Mayor de San Marcos, Lima- Perú.

\section{Análisis Poblacional}

Se determinó la Prevalencia (PREV), Intensidad Media (IM) y Abundancia Media (AM) por pez, por lado derecho e izquierdo y para cada uno de los arcos branquiales con sus respectivas secciones. La longitud estándar de los hospederos se dividieron en seis rangos aproximadamente de $1,8 \mathrm{~cm}$ cada uno, siguinedo la regla de sturges (Zar, 1996). El número de peces hembras fue de 80 y de machos fue de 40. Las hembras presentaron una longitud entre 16,4 $27,0 \mathrm{~cm}$ (promedio $=21,08 \pm 2,24 \mathrm{~cm}$ ). Los machos presentaron una longitud entre 16,5-26,3 $\mathrm{cm}$ (promedio $=20,47 \pm$ $2,06 \mathrm{~cm}$ ). El Coeficiente de Dispersión (CD) fue determinado como la relación entre la Varianza/ IM para cada uno de los dos ectoparásitos.

La prueba de tde Student, previa evaluación de homogeneidad de varianzas empleando la prueba de Levene, fue usada para determinar si la longitud estándar de los peces hospederos machos y hembras presentaban diferencias significativas. La influencia de la talla del hospedero en la PREV de infestación de las dos especies de ectoparásitos se determinó usando el Coeficiente de Correlación de Spearman $\left(\mathrm{r}_{\mathrm{s}}\right)$. Se utilizó el Coeficiente de Correlación de Pearson para determinar la relación del tamaño del hospedero con la IM y AM del cada uno de los dos ectopárasitos. Se aplicó el $\mathrm{c}^{2}$ para tablas de contigencia para determinar el grado de dependencia entre el sexo del hospedero y la PREV de este ectoparásito. El efecto del sexo en la IM y la AM de infestación de ambos parásitos, se calculó utilizando la prueba de $t$ de Student, previo empleo de la prueba de Levene. Se determinó mediante la prueba de $\mathrm{t}$ de Student si existían diferencias en las longitudes estándar de los peces parasitados y no parasitados para cada ectoparásito. Se utilizó nuevamente la prueba de $\mathrm{c}^{2}$ para tablas de contingencia para determinar preferencias en la PREV del parásito a arcos y secciones branquiales en el pez hospedero. Para el cálculo de la preferencia con relación a la IM y AM en los arcos y secciones branquiales se usó el ANDEVA de una vía con un diseño aleatorizado; en el caso de existir diferencias significativas, se utilizó la prueba de Tukey para discrimininar a los posibles arcos y sectores branquiales que estarían causando las diferencias (Zar, 1996). Además se utilizó el $c^{2}$ para determinar si los dos ectoparásitos siguieron la distribución binomial negativa. Se calculó la frecuencia de dominancia individual para cada ectoparásito y para $L$. paralonchuri (hembra y macho) empleando los criterios de Luque \& Chávez (1999). Se usó una matriz de correlación de Spearman entre la abundancia media de cada uno de los cuatro arcos branquiales de $C$. cynoscioni, L. paralonchuri total, macho y hembra. El nivel de significancia fue evaluado a $\mathrm{a}=0,05$.

La terminología ecológica para la PREV, IM y AM siguió los criterios de Bush et al. (1997). Para el cálculo de las pruebas estadísticas descriptivas e inferenciales se usó el paquete estadístico SPSS 12,0 para Windows 98.

\section{RESULTADOS}

La Tabla 1, muestra la PREV, IM y AM de infestación de $C$. cynoscioni y $L$ paralonchuri en los 120 hospederos muestreados. Se observa una distribución sobredispersa o contagiosa solo para $C$. 
cynoscioni, pues el coeficiente de dispersión es mayor a $1(3,13)$ y su preferencia al microhábitat de los filamentos branquiales. Para todos los casos los valores de la PREV, IM, AM y CD de C. cynoscioni fueron mayores que los de $L$. paralonchuri. En el caso de $L$. paralonchuri (macho) $>$ L. paralonchuri (hembra) para la PREV y AM; lo opuesto es para la IM y el CD. La proporción sexual fue machos: hembras $(1: 0,6)$. Cynoscionicola cynoscioni presenta una mayor frecuencia de dominancia en comparación con $L$. paralonchuri. Además ninguno de los dos parásitos siguieron la distribución binomial negativa significativa (Tabla 2; Fig. 1).

El promedio de la longitud de los peces machos $(20,47 \pm 2,06 \mathrm{~cm})$ y de las hembras $(21,08 \pm 2,24 \mathrm{~cm})$ en la muestra analizada, asumiendo igualdad de varianzas no fue significativamente diferente $(t=1,23$, $\mathrm{P}=0,22$ ).

En la mayoría de los casos, la PREV, la IM y la AM de infestación en ambos ectoparásitos no mostraron correlación significativa con la longitud estándar de $C$. analis (Tabla 3 ). A excepción de $C$. cynoscioni que indicó una relación entre la longitud estándar y la AM de infestación y L. paralonchuri (macho) con relación entre la PREV e IM y la longitud estándar.

Con relación al sexo de $C$. analis, los dos ectoparásitos no mostraron diferencias en la PREV, IM y AM entre machos y hembras (Tabla 4).

La Tabla 5, nos indica que solo la IM de $C$. cynoscioni mostró diferencias entre los peces parasitados y los no parasitados.

La Tabla 6, muestra para la PREV preferencias de C. cynoscioni por el I arco y $L$. paralonchuri el IV arco branquial en C. analis. No se observó preferencias en la PREV de ningún ectoparásito por ninguna de las 24 secciones branquiales.

El ANDEVA realizado con relación a la AM en los cuatro arcos branquiales para ambos ectoparásitos, indicó el mismo comportamiento que para la PREV (Tablas 7 a 10). Sin embargo, no se observaron preferencias en la IM y AM para ninguna de las 24 secciones branquiales (Tablas 7 al 10).
La Tabla 11 nos muestra una matriz de correlación de Spearman de la AM branquial entre dos ectoparásitos de $C$. analis. Existe una correlación negativa significativa entre $C$. cynoscioni y $L$. paralonchuri; C. cynoscioni y $L$. paralonchuri (hembra) y entre $L$. paralonchuri (total) y L. paralonchuri (hembra).

\section{DISCUSIÓN}

El presente estudio determinó algunos patrones en la ecología parasitaria de C. cynoscioni y L. paralonchuri, PREV mayor del $10 \%$ y menor del $60 \%$; ausencia del modelo de la distribución binomial negativa en sus hospederos; $y$ en general ausencia de relación entre la PREV, IM y AM de ambos ectoparásitos con la longitud estándar y el sexo de $C$. analis y preferencia a ciertos arcos branquiales por ambos ectoparásitos.

Oliva \& Luque (1998) indican que Cynoscionicola sciaenae Tantaleán, 1974 presentó una PREV e IM de 22,2 \% y 1,3 $\%$ en S. deliciosa $(\mathrm{n}=249)$. Cynoscionicola americana Tantaléan, Martinez \& Escalante, 1987 presentó generalmente altas prevalencias (> 50\%) e IM (> 3) para S. fasciata (78 \% y 5); $P$. peruanus $(78,2 \%$ y 6,1$)$ y $M$. ophicephalus $(52,7 \%$ y 4,2); en cambio bajas para $S$. minor $(0,3 \%$ y 1$)$. En estos casos la prevalencia se encuentró sobre el $20 \%$, semejante a lo encontrado en $C$. cynoscioni (Tabla 1). En cambio para $L$. paralonchuri en $P$. peruanus la PREV e IM fue de $0,8 \%$ y 3 respectivamente. Para Lernanthropus huamani Luque \& Farfán, 1990 su PREV e IM fue respectivamente para $M$. ophicephalus $(80,2 \%$ y 3,1$)$ y para $S$. deliciosa $(1,6 \%$ y 1$)$. En este trabajo se encontró que $L$. paralonchuri una PREV con un valor intermedio $(16,7)$ e IM $(1,26)$ (Tabla 1). La distribución sobredispersa de $C$. cynoscioni $(\mathrm{CD}=3,13)$ cumple la regla existente en procesos parasitarios en peces marinos (Iannacone et al., 2000b). La ausencia de esta distribución sobredispersa en $L$. paralonchuri podría deberse a que presenta infestaciones de baja prevalencia e intensidad (Luque, 1994). Otro copépodo Neobrachiella menticirrhi Luque \& 
Farfán, 1991 mostró este mismo comportamiento en M. ophicephalus (Iannacone, 1991).

Cynoscionicola ha observado patrones variables con relación a la longitud estándar de hospedero y la PREV, IM y AM. Cynoscionicola sciaenae no presentó relación entre la PREV e IM con $S$. deliciosa. $C$. americana en $M$. ophicephalus indicó un patrón de aumento de relación ecológica con su hospedero (Oliva \& Luque, 1998). La presente investigación indicó una relación de $C$. cynoscioni entre la longitud estándar y la AM. Lo cual armoniza con la mayor talla de los peces parasitados con este monogeneo (Tabla 5). (Gutierrez \& Martorelli, 1994; Tuckey \& Joy, 1996).

Para una especie congenérica a $L$. paralonchuri se han detectado patrones variantes con relación la longitud del hospedero y patrones ecológicos. Así, $L$. huamani presentó un aumento de la PREV e IM con M. ophicephalus (Luque, 1994). En nuestro estudio, L. paralonchuri no mostró relación con la longitud de $C$. analis. Sin embargo, el análisis realizado separadamente en este copépodo dioico, en machos y hembras, indica que los peces machos presentó un patrón diferente a las hembras, debido a que los primeros presentan un relación de su PREV e IM con la talla de $C$. analis (Tabla 3). Este patrón ecológico aún no ha sido estudiado en peces marinos peruanos.

El sexo de $C$. analis no demostró influencia en la infestación por ambos ectoparásitos estudiados (Tabla 4). Este comportamiento se ha observado en $C$. americana y L. huamani en $M$. ophicephalus (Iannacone, 1991). La carencia de relación con el sexo en ectoparásitos es atribuido a comportamiento similar entre peces machos y hembras (Tuckey \& Joy, 1996; Brasil-Sato \& Pavanelli, 1989; Luque \& Chavez, 1999; Lo, 1999; Iannacone et al., 2000a, c). Iannacone et al. (2000b) encontraron en el monogeneo Diplectanum sp. en $C$. analis una ausencia de relación con el sexo.

Los patrones de preferencia a un determinado sitio de infestación por los ectoparásitos, es un tópico bien documentado (El Hafidi et al., 1998;
Iannacone et al., 2000b). C. cynoscioni una mayor PREV y AM al I arco branquial y L. paralonchuri al IV arco branquial (Tablas 6 al 10). Especies congenéricas a ambos ectoparásitos muestran patrones variables. Cynoscionicola americana en Menticirrhus ophicephalus no muestra preferencia en su infestación por un determinado arco branquial y $L$. huamani prefiere el II arco branquial. Luque (1994) muestra que los ectoparásitos que prefieren el I y IV arco branquial presentan un mecanismo de ingreso activo a su hospedero, siendo que la corriente de agua es mayor en el II y III arco branquial (Gutierrez \& Martorrelli, 1994). Sin embargo, este mecanismo de ingreso aún no podría aseverarse hasta que los modelos del ciclo de vida de estos ectoparásitos sean bien conocidos (Speare, 1995). Otro factor implicado sería la especificidad morfológica al sustrato, muestran una reducción del tamaño del arco branquial del I al IV. Un tercer factor señalado para explicar la preferencia branquial como la concentración de individuos para facilitar la cópula (Iannacone \& Luque, 1993), podría explicar el comportamiento de infestación de la especie hermafrodita $C$. cynoscioni. Sin embargo, para $L$. paralonchuri al ser dioico, y a pesar de que los especimenes machos y hembras prefieren coincidentemente el IV arco branquial, solo dos peces presentaron este copépodo con especimenes de los dos sexos en un mismo hospedero, observándose mayormente una asociación negativa (Iannacone et al. 2000c). La modificación del hábitat por la presencia de otras especies y evitar procesos competitivos, no sería una explicación satisfactoria en estos dos ectoparásitos de C. analis, pues según Iannacone et al. (2000c) los sectores vacantes alcanzan al $89,87 \%$ de los sectores branquiales, siendo muy improbable una competencia interactiva de diferentes especies de ectoparasitos.

\section{CONCLUSIONES}

1. Cynoscionicola cynoscioni y Lernanthropus paralonchuri tuvieron una distribución poblacional espacial sobre- 
dispersa (3,13 y 0,42 , respectivamente), entre los hospederos evaluados.

2. Se observó una carencia de relación entre la prevalencia, intensidad y abundancia media de os ectoparasitos con la longitud y el sexo de $C$. analis. Solo $C$. cynoscioni mostró una relación positiva entre la abundancia media y el tamaño corporal del pez y L. paralonchuri tuvo la misma relación entre la prevalencia y la intensidad media con la longitud.

3. La mayor prevalencia y el promedio de abundancia de C. cynoscioni y L. paralonchuri se encontró en el I y IV par branquial, respectivamente.

4. Los parasitos no mostraron preferencias a los 24 sectores branquiales (anterior, medio y posterior).

Agradecimientos. Este trabajo fue presentado en el IX Congreso Latinoamericano sobre Ciencias del Mar, San Andrés Isla, Colombia Septiembre 1620 de 2001.

\section{LITERATURA CITADA}

ALBITRES, V. 1965. Relación PesoLongitud y Factor de condición de la denominada Cachema (Cynoscion analis y Cynoscion altipinnis). Tesis para optar el Título de Biólogo Pesquero. Univ. Nac. Trujillo. Perú. $69 \mathrm{pp}$.

BRASIL-SATO, M. C. y PAVANELLI, G.C. 1999. Ecological and reproductive aspects of Neoechinorhynchus pimelodi Brasil-Sato \& Pavanelli ( E o a c a $\mathrm{n} \mathrm{hoce} \mathrm{ph} \mathrm{a} 1$ a, Neoechinorhynchidae) of Pimelodus maculatus Lacépède (Siluroidei, Pimelodidae) of the Sao Francisco River, Brazil. Revista brasileira de Zoologia. 16: 73-82.

BUSH, A. O. y HOLMES, J.C. 1986. Intestinal helminthes of lesser scaup ducks: an interactive community. Canadian Journal of Zoology. 64: 142-152.

BUSH, A. O.; LAFFERTY, K.D.; LOTZ, J.L. \& SHOSTAK, A.W. 1997. Parasitology meets ecology on its own terms: Margolis et al. revisited. Journal of Parasitology 83: 575-583.
CHIRICHIGNO, N. y VELEZ, M. 1998. Clave para identificar los peces marinos del Perú. Pub. Esp. Inst. Mar ( $2^{\mathrm{da}}$ Ed.). 500 pp.

EL HAFIDI, F.; BERRADA-RKHAMI, O.; BENAZZOU, T. y GABRION, C. 1998. Microhabitat distribution and coexistence of Microcotylidae (Monogenea) on the gills of the striped mullet Mugil cephalus: change or competition. Parasitological Research. 84: 315-320.

ESTRELLA, C.y GUEVARACARRASCO, R. 1977. Informe estadístico anual de los recursos hidrobiológicos de la pesquería artesanal por especies, artes, caletas y meses durante 1997. Informes del Instituto del Mar del Perú. 131: 1-222.

GEORGE-NASCIMENTO, M. y IRIARTE， J.L. 1989. Las infracomunidades de parásitos metazoos del chancharro Helicolenus lengerichi Norman, 1937 (Pisces: Scorpaenidae): un ensamble no interactivo de especies. Revista Chilena de Historia Natural. 62: 217227.

GUTIERREZ, P. A. y MARTORELLI, S.R. 1994. Seasonality, distribution, and preference sites of Demidospermus valenciennesi Gutierrez et Suriano, 1992 (Monogenea: Ancyrocephalidae) in catfish. Research Reviews of Parasitology 54: 259-261.

HAYWARD, C. J.; PERERA, K.M. y ROHDE, K. 1998. Assemblages of ectoparasites of a pelagic fish, slimy mackerel (Scomber australasicus), from south-eastern Australia. International Journal for Parasitology. 28: 263-273.

IANNACONE, J. 1991. Dinámica poblacional de la fauna parasitaria (Metazoa) de Menticirrhus ophicephalus (Pisces: Sciaenidae) de la costa central peruana. Tesis Licenciado en Biología. Univ. Ricardo Palma, Lima, Perú. 85 p.

IANNACONE, J. y LUQUE, J.L. 1993. Aspectos ecológicos de los parásitos branquiales del bagre, Galeichthyis peruanus (L.) (Pisces: Teleostei) en la Costa Central del Perú. Boletín de Lima (Perú). 88: 69-73. 
IANNACONE, J. A.; LÓPEZ, E.N. y ALVARIÑO, L.F. 2000a. Procamallanus (Spirocamallanus) inopinatus Travassos, Artigas et Pereira, 1928 (Nematoda: Camallanidae) endoparásito de Triportheus angulatus (Spix, 1829) (Characidae) en la laguna de Yarinacocha, UcayaliPerú. Biología Pesquera (Chile). 28: 37-43.

IANNACONE, J.; MEJÍA, W.; ALCOCER, F.; BRIONES, G. y ROMÁN, A. 2000b. Características de la infestación de Diplectanum sp. (Monogenea: Monopisthocotylea: Diplectanidae) en el ayanque Cynoscion analis Jenyns (Pisces: Teleostei: Sciaenidae). Revista Peruana de Biología 7: 44-54.

IANNACONE, J.; TATAJE, J.; FUENTES-RIVERA, J.; ÁLVAREZ, K. y AGUILAR, P. 2000c. Infracomunidades ectoparasitarias en las branquias de la cachema Cynoscion analis Jenyns (Pisces: Sciaenidae). Revista Peruana de Parasitología. 15: 42-54.

IANNACONE, J. 2004. Metazoos parásitos de la mojarrilla Stellifer minor (Tschudi) (Osteichthyes, Sciaenidae) capturados por pesquería artesanal en Chorrillos, Lima, Perú. Revista Brasileira de Zoologia. 21: 815-820.

KRITSKY, D.; THATCHER, V. y BOEGER, W. 1986. Neotropical Monogenea. 8. Revisión of Urocleidoides (Dactylogyridae, Ancyrocephalinae). Proceedings of the Helminthological Society of Washington.53: 1-37.

LO, C. M. 1999. Mating rendezvous in monogenean gill parasites of the humbug Dascyllus aruanus (Pisces: Pomacentridae). Journal of Parasitology. 85: 1178-1180.

LUQUE, J. L. 1994. Dinámica poblacional y estructura de la comunidad de metazoarios parásitos de Menticirrhus ophicephalus (Pisces: Sciaenidae) de la costa peruana. Revista Biología Tropical. 42: 21-29.

LUQUE J. L. 1996. Distribución transversal y asociaciones interespecífica en las comunidades de metazoarios ectoparásitos de peces esciénidos marinos del Perú. Revista Biología Tropical. 44: 383.390.

LUQUE, J. L. y CHAVES, N.D. 1999. Ecología da comunidade de metazoarios parasitos da anchova Pomatomus saltator (Linnaeus) (Osteichthyes, Pomatomidae) do litoral do estado do Rio de Janeiro, brasil. Revista brasileira de Zoologia. 16: 711-723.

LUQUE, J. L. y FARFÁN, C. 1991. Caligus quadratus (Shiino, 1954) (Copepoda: Caligidae) ectoparásito de peces marinos del Perú. Boletín de Lima (Perú). 78: 81-86.

LUQUE, J. L. y OLIVA, M.E. 1993. Análisis cuantitativo y estructura de la comunidad parasitaria de Paralonchurus peruanus (Pisces: Sciaenidae) en la costa peruana. Parasitología al Día. 17: 107-111.

LUQUE, J. L.; IANNACONE, J. y FARFÁN, C. 1991. Parásitos de peces óseos marinos en el Perú: lista de especies conocidas. Boletín de Lima (Perú). 74: 17-28.

MENDO, J.; SAMAMÉ, M; WOSNITZAMENDO, A.; MENDIETA, J. y CASTILlO, C. 1988. Análisis biológico-pesquero y poblacional de la cachema (Cynoscion analis) del área de Paita Perú. Boletín del Instituto del Mar del Perú. 12: 1-20.

OLIVA, M. E. y LUQUE, J.L. 1998. Metazoan parasites infracommunities in five sciaenids from the Central Peruvian Coast. Memorias do Instituto Oswaldo Cruz. 93: 175-180.

OLIVA, M. E.; LUQUE, J.L. y IANNACONE, J. 1989. Prevalencia y patrones de distribución de tres especies de monogeneos en las branquias de Stellifer minor (Tschudi, 1844) (Osteichthyes: Sciaenidae). Revista Ibérica de Parasitología. 49: 209-214.

OLIVA, M. E.; LUQUE, J.L. y IANNACONE, J. 1990. The metazoan parasites of Stellifer minor (Tschudi, 1844): An ecological approach. Memorias do Instituto Oswaldo Cruz. 85:271-274. 
SALDARRIAGA, C. 1977. La parasitosis y su relación con el factor de condición, sexo y la longitud de la «cachema» Cynoscion analis. Tesis Bachiller en Ciencias Biológicas. Univ. Nac. Trujillo. Perú.

SPEARE, P. 1995. Parasites as biological tags for sailfish Istiophorus platypterus from east coast Australian waters. Marine Ecology Progress Series 118: 43-50.

TUCKER, R. B. y JOY, J.E. 1996. Relationships between Glypthelmins pennsylvaniensis (Trematoda:
Digenea) infections and host size. Journal of the Helminthological Society of Washington. 63: 42-46.

WILLIAMS, A. 1989. Some monogenean parasites of the genera Calceostoma Van Beneden, 1852 and Diplectanum Diesing, 1858 from Argyrosomus hololepidotus (Lacépede, 1802) (Sciaenidae: Teleostei) in Western Australia. Systematic Parasitology. 14: 187-201.

ZAR, J. H. 1996. Biostatistical Analysis. $3^{\text {th }}$ Ed. Prentice-Hall, Inc. Upper Saddle River. New Yersey. 662 pp. 
Tabla 1. Prevalencia, intensidad y abundancia de infestación de dos ectoparásitos de Cynoscion analis en el Terminal Pesquero de Chorrillos, Lima, Perú.

\begin{tabular}{|c|c|c|c|c|}
\hline Parásitos & $\begin{array}{c}\text { Prevalencia } \\
(\%)\end{array}$ & $\begin{array}{l}\text { Intensidad } \\
\text { media } \\
\text { (IM) }\end{array}$ & $\begin{array}{l}\text { Abundancia } \\
\text { media } \\
(\text { AM) }\end{array}$ & $\begin{array}{r}\text { Coeficiente } \\
\text { dispersión } \\
\text { (CD) }\end{array}$ \\
\hline \multicolumn{5}{|l|}{ Monogenea } \\
\hline Cynoscionicola cynoscioni & 21,7 & $1,84 \pm 2,38$ & $0,39 \pm 1,32$ & 3,13 \\
\hline \multicolumn{5}{|l|}{ Copepoda } \\
\hline Lernanthropus paralonchuri $*$ & 16,7 & $1,26 \pm 0,73$ & $0,20 \pm 0,54$ & 0,42 \\
\hline Lernanthropus paralonchuri ${ }^{(1)}$ & 10,83 & $1,15 \pm 0,38$ & $0,12 \pm 0,37$ & 0,12 \\
\hline Lernanthropus paralonchuri ${ }^{(2)}$ & 5,83 & $1,29 \pm 0,49$ & $0,07 \pm 0,32$ & 0,19 \\
\hline
\end{tabular}

Tabla 2. Frecuencia de dominancia y valores de $X^{2}$ para la distribución binomial negativa de dos parásitos de Cynoscion analis en el Terminal Pesquero de Chorrillos, Lima, Perú.

Parásitos
Frecuencia de dominancia
Frecuencia de dominancia compartida entre dos especies

Monogenea

Cynoscionicola cynoscioni

Copepoda

Lernanthropus paralonchuri *

Lernanthropus paralonchuri

Lernanthropus paralonchuri ${ }^{(2)}$

$*=$ total.

(1) $=$ macho

${ }^{(2)}=$ hembra.

\begin{tabular}{rrrr}
4 & 6 & 19,14 & $>0,05$ \\
3 & 3 & 21,48 & $>0,05$ \\
2 & 3 & 5,68 & $>0,05$ \\
\hline
\end{tabular}

Tabla 3. Valores de correlación de Pearson (r) entre la Prevalencia (PREV), intensidad media (IM) y al abundancia media (AM) de infestación de dos parásitos con la longitud estándar de Cynoscion analis en el Terminal Pesquero de Chorrillos, Lima, Perú. $(\mathbf{P}=$ Probabilidad $)$

\begin{tabular}{|c|c|c|c|c|c|c|}
\hline \multirow[b]{2}{*}{ Parásitos r } & \multicolumn{2}{|c|}{ PREV } & \multicolumn{2}{|c|}{ IM } & \multicolumn{2}{|c|}{$\mathbf{A M}$} \\
\hline & $\mathbf{r}$ & $\mathbf{P}$ & $\mathbf{r}$ & $\mathbf{P}$ & $\mathbf{r}$ & $\mathbf{P}$ \\
\hline \multicolumn{7}{|l|}{ Monogenea } \\
\hline Cynoscionicola cynoscioni & 0,91 & 0,10 & 0,19 & 0,35 & 0,92 & 0,01 \\
\hline \multicolumn{7}{|l|}{ Copepoda } \\
\hline Lernanthropus paralonchuri * & 0,11 & 0,53 & 0,19 & 0,31 & 0,02 & 0,83 \\
\hline Lernanthropus paralonchuri (1) & 0,86 & 0,03 & 0,57 & 0,04 & 0,09 & 0,31 \\
\hline Lernanthropus paralonchuri ${ }^{(2)}$ & 0,37 & 0,46 & 0,25 & 0,58 & $-0,07$ & 0,40 \\
\hline
\end{tabular}


Tabla 4. Valores de prueba de t de Student para la Prevalencia (PREV), intensidad media (IM) y al abundancia media (AM) de infestación de dos parásitos con el sexo de Cynoscion analis en el Terminal Pesquero de Chorrillos, Lima, Perú. $(\mathbf{P}=$ Probabilidad $)$

\begin{tabular}{lcccccc} 
& \multicolumn{2}{c}{ PREV } & \multicolumn{2}{c}{ IM } & \multicolumn{2}{c}{ A M } \\
\cline { 2 - 7 } Parásitos & $\boldsymbol{X}^{\mathbf{2}}$ & $\mathbf{P}$ & $\mathbf{t}$ & $\mathbf{P}$ & $\mathbf{t}$ & $\mathbf{P}$ \\
\hline $\begin{array}{l}\text { Monogenea } \\
\text { Cynoscionicola cynoscioni }\end{array}$ & 2,58 & $>0,05$ & 0,87 & 0,39 & 1,25 & 0,21 \\
\hline $\begin{array}{l}\text { Copepoda } \\
\text { Lernanthropus paralonchuri }^{*}\end{array}$ & 1,92 & $>0,05$ & 1,02 & 0,32 & 1,46 & 0,15 \\
Lernanthropus paralonchuri $^{(1)}$ & 2,60 & $>0,05$ & 1,43 & 0,18 & 0,68 & 0,09 \\
Lernanthropus paralonchuri $^{(2)}$ & 0,09 & $>0,05$ & 0,21 & 0,85 & 0,55 & 0,58 \\
\hline
\end{tabular}

* = total.

(1) = macho.

(2) $=$ hembra

Tabla 5. Longitudes estándares de $C$. analis infectados con cada uno de los dos ectoparásitos en comparación con los no infectados en el Terminal Pesquero de Chorrillos, Lima, Perú.

\begin{tabular}{lcccccc}
\multicolumn{1}{c}{ Ectoparásito } & $\mathrm{Ni}$ & Promedio $( \pm \mathrm{DE})$ & $\mathrm{Nn}$ & Promedion $( \pm \mathrm{DE})$ & $\mathrm{t}$ & $\mathrm{P}$ \\
\hline C. cynoscioni & 26 & $21,81(2,09)$ & 94 & $20,73(2,09)$ & 2,33 & 0,02 \\
L. paralonchuri (total) & 19 & $20,69(2,26)$ & 101 & $21,02(2,11)$ & 0,61 & 0,54 \\
L. paralonchuri (macho) & 13 & $21,13(2,54)$ & 107 & $20,94(2,09)$ & 0,28 & 0,77 \\
L. paralonchuri (hembra) & 7 & $20,14(1,51)$ & 113 & $21,02(2,16)$ & 1,06 & 0,29 \\
\hline
\end{tabular}

$\mathrm{Ni}=$ Número de hospederos infectados. $\mathrm{DE}=$ Desviación estándar. $\mathrm{Nn}=$ Número de hospederos no infectados. $\mathrm{i}=$ infectados $\mathrm{n}=$ no infectados

Tabla 6. Prueba de Chi-cuadrado para la preferencia branquial en la Prevalencia de dos ectoparásitos en Cynoscion analis en el Terminal Pesquero de Chorrillos, Lima, Perú

\begin{tabular}{llll}
\multirow{2}{*}{ ECTOPARÁSITO } & \multicolumn{1}{c}{ AI } & \multicolumn{1}{c}{ AD } & \multicolumn{1}{c}{ A Total } \\
\cline { 2 - 4 } & \multicolumn{1}{c}{$X^{2}$} & \multicolumn{1}{c}{$X^{2}$} & \multicolumn{1}{c}{$X^{2}$} \\
\hline Cynoscionicola cynoscioni $^{*}$ & $13,45^{* *}(\mathrm{I})$ & 4,82 & $28,93^{* *}(\mathrm{I})$ \\
Lernanthropus paralonchuri $^{*}$ & $46,97^{* *}(\mathrm{IV})$ & $21,72^{* *}(\mathrm{IV})$ & $38,77^{* *}(\mathrm{IV})$ \\
Lernanthropus paralonchuri $^{(1)}$ & $14,14^{* *}(\mathrm{IV})$ & $21,8^{* *}(\mathrm{IV})$ & $12,82^{* *}(\mathrm{IV})$ \\
Lernanthropus paralonchuri $^{(2)}$ & $11,19^{* *}(\mathrm{IV})$ & $11,19^{* *}(\mathrm{IV})$ & $19,73 * *(\mathrm{IV})$ \\
\hline
\end{tabular}

$c^{2}$ tabulado $=7,81$ para los arcos branquiales.

$* *$ Valores significativos.

Los paréntesis indican los arcos branquiales de mayor prevalencia.

$\begin{array}{ll}* & \text { (1) } \\ & =\text { macho. }\end{array} \quad{ }^{(2)}=$ hembra. $\quad \mathrm{AI}=$ Arco Izquierdo. $\mathrm{AD}=$ Arco Derecho. 
Tabla 7. Valores del estadístico $F$ y su nivel de significancia (P) de la intensidad media y abundancia media branquial de Cynoscionicola cynoscioni en Cynoscion analis.

\begin{tabular}{lcccc} 
& \multicolumn{2}{c}{ Intensidad Media } & \multicolumn{2}{c}{ Abundancia Media } \\
\cline { 2 - 5 } ARCO BRANQUIAL & $\mathbf{F}$ & $\mathbf{P}$ & $\mathbf{F}$ & $\mathbf{P}$ \\
\hline Área Izquierda & 0,88 & 0,47 & 1,50 & 0,21 \\
Área Derecha & 1,07 & 0,37 & 3,73 & $0,02 *(\mathrm{I}=0,11)$ \\
Área Total & 0,36 & 0,78 & 3,72 & $0,02 *(\mathrm{I}=0,17)$ \\
SECCIÓN BRANQUIAL & $\mathbf{F}$ & $\mathbf{P}$ & $\mathbf{F}$ & $\mathbf{P}$ \\
Área Izquierda & 1,42 & 0,27 & 0,15 & 0,86 \\
Área Derecha & 0,32 & 0,73 & 0,59 & 0,55 \\
Área Total & 1,16 & 0,33 & 0,54 & 0,58 \\
\hline
\end{tabular}

* = Significativo y arcos branquiales con mayor infestación.

Tabla 8. Valores del estadístico $F$ y su nivel de significancia (P) de la intensidad media y abundancia media branquial de Lernanthropus paralonchuri en Cynoscion analis.

\begin{tabular}{lcccc} 
& \multicolumn{2}{c}{ Intensidad Media } & \multicolumn{2}{c}{ Abundancia Media } \\
\cline { 2 - 5 } ARCO BRANQUIAL & $\mathbf{F}$ & $\mathbf{P}$ & $\mathbf{F}$ & $\mathbf{P}$ \\
\hline Área Izquierda & 0,81 & 0,47 & 4,12 & $0,007^{*}(\mathrm{IV}=0,008)$ \\
Área Derecha & $-* *$ & - & 7,78 & $<0,001^{*}(\mathrm{IV}=0,07)$ \\
Área Total & 0,09 & 0,91 & 15,46 & $<0.001^{*}(\mathrm{IV}=0,175)$ \\
SECCIÓN BRANQUIAL & $\mathbf{F}$ & $\mathbf{P}$ & $\mathbf{F}$ & $\mathbf{P}$ \\
Área Izquierda & 0,92 & 0,43 & 0,44 & 0,65 \\
Área Derecha & - & - & 0,34 & 0,71 \\
Área Total & 0,68 & 0,51 & 0,73 & 0,48 \\
\hline
\end{tabular}

* =Significativo y arco branquial con mayor infestación.

** = Indica que al menos dos arcos branquiales no presentaron ningún parásito, por lo que el ANVA no se pudo realizar.

Tabla 9. Valores del estadístico $F$ y su nivel de significancia (P) de la intensidad media y abundancia media branquial de Lernanthropus paralonchuri (macho) en Cynoscion analis.

\begin{tabular}{llccc} 
& \multicolumn{2}{c}{ Intensidad Media } & \multicolumn{2}{c}{ Abundancia Media } \\
\cline { 2 - 5 } ARCO BRANQUIAL & $\mathbf{F}$ & $\mathbf{P}$ & $\mathbf{F}$ & $\mathbf{P}$ \\
\hline Área Izquierda & 1,00 & 0,35 & 5,17 & $<0,002^{*}(\mathrm{IV}=0,05)$ \\
Área Derecha & $-* *$ & - & 3,32 & $<0,002(\mathrm{IV}=0,04)$ \\
Área Total & - & - & 7,78 & $<0,001 *(\mathrm{IV}=0,10)$ \\
SECCIÓN BRANQUIAL & $\mathbf{F}$ & $\mathbf{P}$ & $\mathbf{F}$ & $\mathbf{P}$ \\
\hline Área Izquierda & 0,56 & 0,60 & 0,81 & 0,44 \\
Área Derecha & - & - & 0,59 & 0,55 \\
Área Total & 0,44 & 0,66 & 1,50 & 0,22 \\
\hline
\end{tabular}

$*$ = Significativo y arcos branquiales de mayor infestación.

** = Indica que al menos dos arcos branquiales no presentaron ningún parásito, por lo que el ANVA no se pudo realizar. 
Tabla 10. Valores del estadístico $F$ y su nivel de significancia $(P)$ de la intensidad media y abundancia media branquial de Lernanthropus paralonchuri (hembra) en Cynoscion analis.

\begin{tabular}{lcccc} 
& \multicolumn{2}{c}{ Intensidad Media } & \multicolumn{2}{c}{ Abundancia Media } \\
\cline { 2 - 5 } ARCO BRANQUIAL & $\mathbf{F}$ & $\mathbf{P}$ & $\mathbf{F}$ & $\mathbf{P}$ \\
\hline Área Izquierda & - & - & 2,71 & $0,04^{*}(\mathrm{IV}=0,04)$ \\
Área Derecha & - & - & 3,65 & $0,01 *(\mathrm{IV}=0,03)$ \\
Área Total & - & - & 6,52 & $<0,001^{*}(\mathrm{IV}=0,07)$ \\
SECCIÓN BRANQUIAL & $\mathbf{F}$ & $\mathbf{P}$ & $\mathbf{F}$ & $\mathbf{P}$ \\
Área Izquierda & - & - & 0,20 & 0,85 \\
Área Derecha & - & - & 1,77 & 0,17 \\
Área Total & - & - & 1,02 & 0,36
\end{tabular}

* = Significativo y arcos branquiales de mayor infestación.

** = Indica que al menos dos arcos branquiales no presentaron ningún parásito, por lo que el ANVA no se pudo realizar.

Tabla 11. Matriz de correlación de Spearman entre la abundancia media branquial de los ectoparásitos de $C$. analis del Terminal Pesquero de Chorrillos, Lima, Perú.

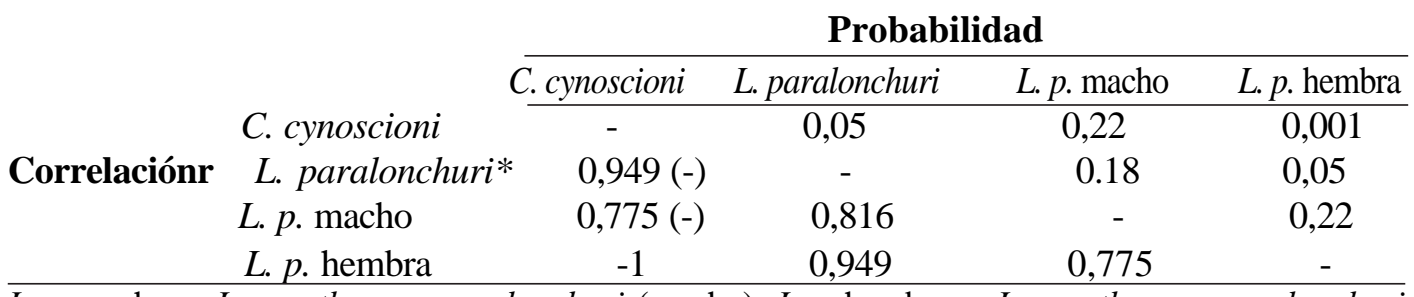

L.p. macho $=$ Lernanthropus paralonchuri $($ macho). L.p. hembra $=$ Lernanthropus paralonchuri (hembra). * totales.

Figura 1. Frecuencia de dos ectoparásitos branquiales de $C$. analis en el Terminal Pesquero de Chorrillos, Lima, Perú.(Footnotes)

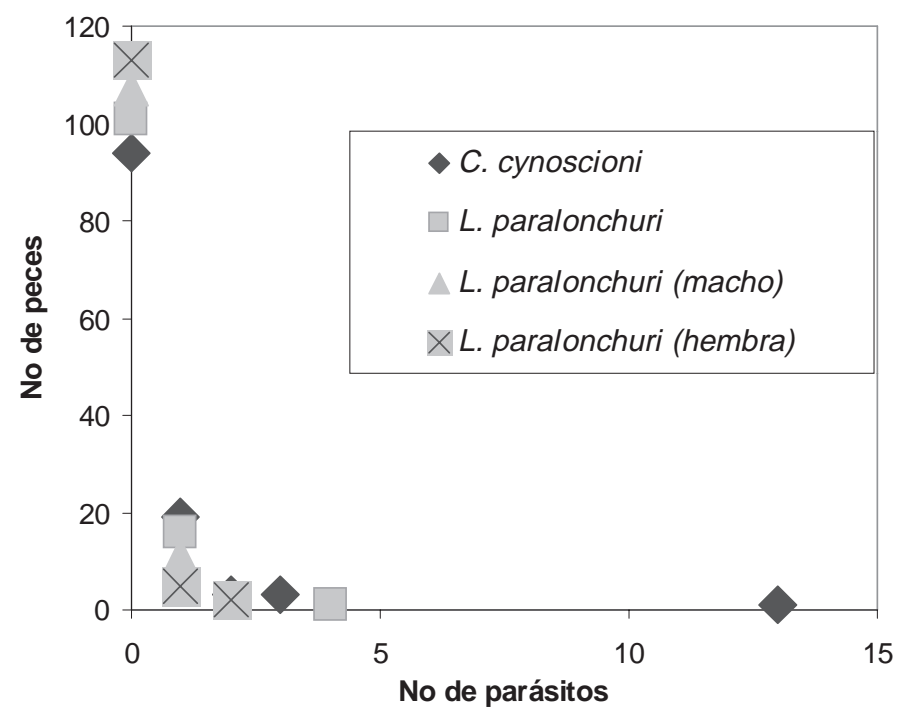

